Nervenarzt 2020 $91: 312-317$

https://doi.org/10.1007/s00115-020-00872-6

Online publiziert: 19. Februar 2020

(c) Der/die Autor(en) 2020

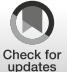

E. Feneberg ${ }^{1,2} \cdot \mathrm{M}$ Otto $^{1}$

${ }^{1}$ Abteilung für Neurologie, Universitäts- und Rehabilitationskliniken, Ulm, Deutschland

${ }^{2}$ Nuffield Department of Clinical Neurosciences, University of Oxford, Level 6, West Wing, John Radcliffe Hospital, Oxford, Großbritannien

\title{
Genspezifische Therapieansätze bei der Alzheimer-Krankheit und anderen Tauopathien
}

ken Einschränkung der Alltagsfunktionen und zur Pflegebedürftigkeit. Pathologisch kommt es bei der AlzheimerKrankheit zur Hyperphosphorylierung von Protein Tau und intrazellulärer Fibrillenbildung, den sog. neurofibrillären Tangles, die zu einer fortschreitenden Degeneration von Nervenzellen führen. Neuropathologisch lässt sich die Alzheimer-Krankheit je nach Ausbreitung der neurofibrillären Tangles in verschiedene Stadien einteilen, die topgraphisch und zeitlich mit dem Auftreten der entsprechenden kognitiven Defizite korrelieren [2].

Hinzu kommen extrazelluläre Amyloid- $\beta$-Ablagerungen. Diese entstehen durch alternative proteolytische Abbauprozesse des Amyloidvorläuferproteins durch $\beta$ - und $\gamma$-Sekretasen, wodurch es $\mathrm{zu}$ einem Missverhältnis zwischen löslichem a-geschnittenen Amyloidvorläuferprotein und Amyloid- $\beta$ kommt. Letzteres wird nun vermehrt gebildet, was zur Entstehung extrazellulärer Amyloidfibrillen führt. Vor allem A $\beta 1-42$ Fragmente neigen im Gegensatz $\mathrm{zu}$ weniger toxischen $A \beta 1-40$-Fragmenten zur Aggregatbildung. Es gibt eine ausreichende Evidenz, dass Amyloid- $\beta$ neurotoxisch ist und negativen Einfluss auf die Tauopathie hat [27]. Dafür spricht auch, dass bisher alle Mutationen, die zur Entwicklung einer familiären Alzheimer-Krankheit führen, den Amyloid$\beta$-Metabolismus beeinflussen. Jedoch ist die monogen, autosomal-dominante vererbte Alzheimer-Krankheit selten. Sie tritt nur in $<1 \%$ der Fälle auf und manifestiert sich meist vor dem 65 . Le- bensjahr („early-onset“), während die meisten Fälle der Alzheimer-Krankheit multifaktoriell bedingt sind und nach dem 65. Lebensjahr auftreten („lateonset").

\section{Antikörpertherapie}

Ausgehend von der Hypothese, dass Amyloid- $\beta$-Aggregate das primäre neurotoxische Agens sind, zielten sog. Immunisierungsstudien darauf $\mathrm{ab}$, das Amyloid- $\beta$ mittels gegen physiologisch und pathologisch veränderte Amyloid- $\beta$-Formen gerichteter monoklonaler Antikörper zu neutralisieren, wodurch dieses abgebaut wird. [9, 24, 25]. PhaseIII-Antikörperstudien gegen Amyloid$\beta$ im Stadium der mainfesten Demenz haben bisher nicht zum erwünschten Erfolg, nämlich einer Verbesserung der kognitiven Leistung geführt, weshalb deren Wirksamkeit hinterfragt wurde. Auch die jüngste Studie wurde aufgrund einer negativen Interimsanalyse und mangelnder Erfolgsaussicht nicht fortgeführt, jedoch konnten die eingeschlossenen Patienten die Einnahme des Studienpräparates auf freiwilliger Basis im Rahmen einer Open-label-Studie fortführen. Eine kürzlich vorgestellte Auswertung auf Basis der hinzugekommenen Datenpunkte bescheinigt der höchsten Dosis des Präparats nun doch einen geringen Einfluss auf den kognitiven Status [1].

Angesichts der insgesamt dennoch ernüchternden Ergebnisse der Immunisierungsstudien wird nun insbesondere diskutiert, ob sich die ursprünglich 
Hier steht eine Anzeige.

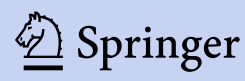




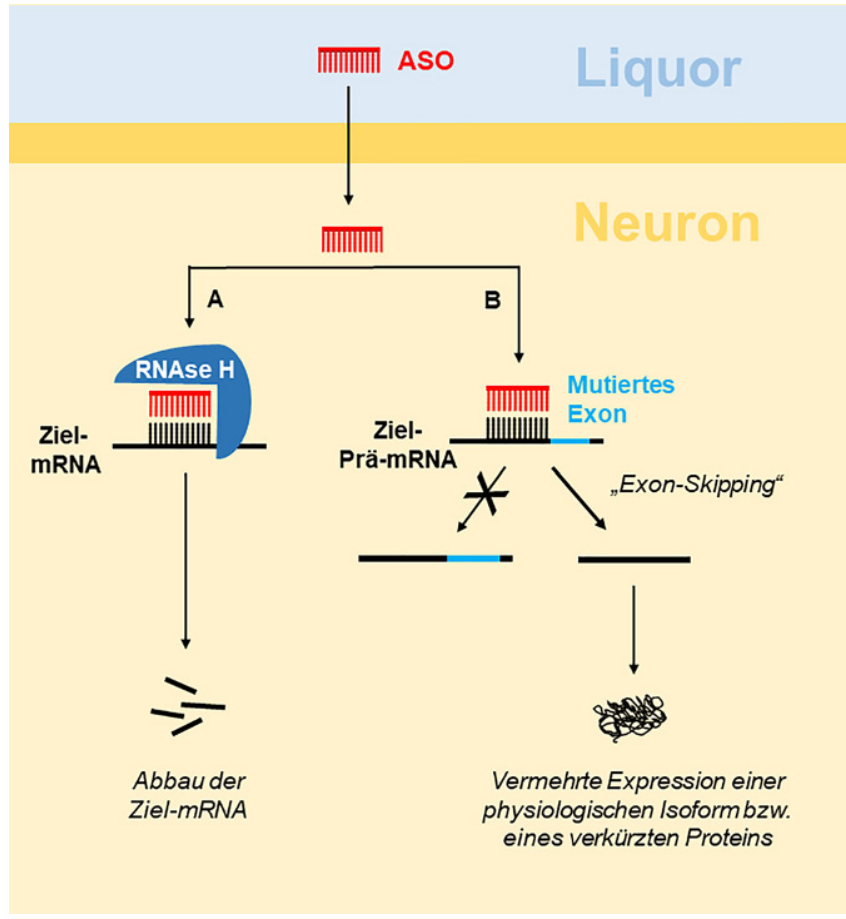

Abb. $1<$ Antisense-Oligonukleotide(ASO)-basierte Therapiestrategien bei Alzheimer-Krankheit und Tauopathien. (A) Bindung des ASO an die Ziel-mRNA führt zum RNAse-Hvermittelten $\mathrm{Abbau}$ der Ziel-mRNA. (B) Bindung des ASO an die Ziel-RNA führt zur Exklusion (Skipping) eines Exons und zur vermehrten Translation einer (verkürzten) Isoform des Proteins von der Amyloid- $\beta$-Proteinopathie ausgehende pathophysiologische Kaskade (u. a. die Tauopathie) im moderat schweren Stadium der Alzheimer-Krankheit bereits zu weit von der Amyloid- $\beta$-Pathologie entkoppelt hat, als dass eine Neutralisierung von Amyloid- $\beta$ noch einen relevanten Einfluss auf die weitere Krankheitsprogression hätte [12]. Daher werden die Folgestudien nun einerseits bei Patienten in früheren Krankheitsphasen und andererseits mit höheren Dosierungen und längerer Studiendauer durchgeführt.

\section{》) Aktuelle Studien untersuchen die Wirkung von Tau-Antikörpern bei $\mathrm{MCl}$ und leichter $\mathrm{AD}$}

Da die Ausbreitung der Tauopathie bei $\mathrm{AD}$ besser mit der Neurodegeneration zu korrelieren scheint als die Amyloid$\beta$-Proteinopathie [2], ruhen die Hoffnungen nun auf den Tau-Antikörpern. Diese werden aktuell - entweder im Rahmen einer aktiven oder passiven Immunisierung - in klinischen PhaseII-Studien bei Patienten mit ,mild cognitive impairment" (MCI) und leichter Alzheimer-Demenz untersucht (AbbVie
C2N-8E12, BIIB092, RO7105705, Axon: NCT02579252; [20, 21]).

\section{Genspezifische Therapieansätze}

Auch bei der AD-Forschung werden Therapieansätze entwickelt, die genspezifisch auf die Korrektur oder die Kompensation zugrunde liegender Genveränderungen oder Pathomechanismen abzielen.

\section{APP}

Bereits 1987 wurde herausgefunden, dass das Amyloid-Precursor-Protein (APP)Gen auf dem Chromosom 21 lokalisiert ist. [15, 28]. Dies erklärte, warum Trisomie-21-Patienten früh an einer Demenz erkranken, da durch das doppelte Vorkommen von APP mehr Amyloid$\beta$ produziert wird und es zur Akkumulation von neurotoxischem Amyloid$\beta$ kommt. Später wurden Mutationen im APP-Gen beschrieben, die zu einer familiären Alzheimer-Krankheit führen [4]. Derzeit sind 39 Mutationen bekannt, die sich auf die vermehrte durch $\beta$ - and $\gamma$-Sekretasen-vermittelte Bildung von APP und Amyloid- $\beta$ auswirken und damit ähnlich der Trisomie 21 zu erhöhten Mengen an Amyloid- $\beta$ führen.
Wie wichtig das $A P P$-Gen für die Alzheimer-Krankheit ist, konnte anhand der isländischen Bevölkerung gezeigt werden, wo eine protektive Variante im APPGen gefunden wurde, die die abnorme Proteolyse von APP verhindert, wodurch weniger $A \beta 1-40$ - und $A \beta 1-42$ Fragmente gebildet werden [14]. Zudem ist bekannt, dass das Ausmaß an Amyloid- $\beta, A \beta 1-40$ und $A \beta 1-42$ negativ mit der Gedächtnisleistung korreliert und dass dies unabhängig von den neurofibrillären Tangles ist [18]. Folglich wurden ASOs entwickelt, die gegen die Amyloid- $\beta$-Region der APP-RNA gerichtet sind und RNAse-H-vermittelt zu deren Abbau führen, wodurch weniger Amyloid- $\beta$ entsteht (• Abb. 1A).

\section{》) Durch ein spezielles ASO kann das C-terminale amyloidogene APP reduziert werden}

Mehrere solcher ASOs wurden präklinisch im SAMP8 („senescence-accelerated prone mouse") -Mausmodell getestet. Bei diesen Mäusen kommt es infolge einer vermehrten Produktion von APP und Amyloid- $\beta$ zu einer frühzeitigen Alterung mit Gedächtnisdefiziten. Dabei führte nur ein ASO, das spezifisch gegen die Amyloid- $\beta$-Region von APP (Aminosäuren 17-30) gerichtet war, nach mehrfacher intrathekaler Verabreichung zu einer Verbesserung der Gedächtnisleistung [16]. Nachweislich reduzierte das ASO das C-terminale amyloidogene APP, während das N-terminale Ende von APP, gemessen mit einem APP-Antikörper gegen die Aminosäuren 8-17 gerichtet, unverändert vorlag. Da die A $\beta 1-40-$ und A $\beta 1$-42-Fragmente aus dem C-terminalen Ende von APP hervorgehen, könnte dieser Ansatz vielversprechend sein, um eine Amyloid- $\beta$-spezifische Reduktion von APP zu erreichen. Da das N-terminale Ende unverändert blieb, sind Offtarget-Effekte innerhalb von APP eher nicht zu erwarten.

In einer Folgestudie wurde der Befund mit einer intravenösen Applikation bestätigt, sodass eine Überschreitung der Blut-Hirn-Schranke und aktive intrazelluläre Aufnahme angenommen werden kann. In dieser Studie wurde gezeigt, dass 
Amyloid- $\beta$-spezifisches APP um $30 \%$ reduziert wurde, allerdings ergaben sich unveränderte Werte für $\mathrm{A} \beta 1-40$, während $A \beta 1-42$ nicht gemessen wurde. $\mathrm{Zu}$ sätzlich konnte in dieser Studie ein Effekt des ASO auf den Amyloid- $\beta$-Transporter LRP-1 gezeigt werden, der für die Endozytose und intrazelluläre Prozessierung zu Amyloid- $\beta$ verantwortlich ist und dessen Konzentration unter ASO-Applikation abnahm [10].

Dasselbe ASO wurde in transgenen APP-Mäusen (Tg2576) getestet, die durch eine Mutation im APP-Gen an Alzheimer erkranken. Hier wurde gezeigt, dass es zur vermehrten Bildung von löslichem $A \beta 1-40$ und zu einer Verbesserung der Gedächtnisleistung auch nach intravenöser Applikation kam [11]. Weiterhin wurde eine Reduktion neuroinflammatorischer Marker gemessen, was neben einem positiven Behandlungseffekt auch für die Sicherheit des ASO relevant ist, deren chemische Struktur proinflammatorische Auswirkungen haben kann.

Eine weitere ASO-Strategie ist die Modulierung von Spleißvorgängen. Das Spleißen ermöglicht, dass aus demselben genetischen Code verschiedene Proteinisoformen entstehen. Durch die Aktivierung von Verstärker- oder Unterdrückerregionen wird das Spleißen von Exonabschnitten der Prä-mRNA moduliert und entsprechend reife mRNA gebildet, die dann die jeweiligen Proteinisoformen kodiert. Kommt es durch ein ASO zur kompetitiven Bindung an einer Verstärkerregionen, kann das Spleißen eines Exons unterdrückt werden (ExonSkipping) und somit die Translation einer spezifischen Proteinregion gehemmt werden.

Dieser Mechanismus wurde genutzt, um das Exon 17 aus der mRNA von $A P P$ auszuschließen und eine verkürzte $A P P$ Variante herzustellen [3]. Durch die fehlende Exon-17-Region kann APP nicht durch die $\gamma$-Sekretase geschnitten werden, wodurch auch die Bildung von Amyloid- $\beta$ (A $\beta 1-42)$ verhindert wird. Diese ASOs werden „splice-switching antisense oligonucleotide“ (SSO) genannt und es konnte in einem ersten Tiermodell gezeigt werden, dass die Entstehung

Nervenarzt 2020 -91:312-317 https://doi.org/10.1007/s00115-020-00872-6

(c) Der/die Autor(en) 2020

\section{E. Feneberg $\cdot$ M. Otto}

\section{Genspezifische Therapieansätze bei der Alzheimer-Krankheit und anderen Tauopathien}

\section{Zusammenfassung}

Die Alzheimer-Krankheit ist pathologisch durch die Aggregation und Akkumulation von Amyloid- $\beta$ und Protein Tau gekennzeichnet. Bisher war das Ziel der meisten Interventionsstudien die toxischen Proteinprodukte zum Beispiel durch die Immunisierung mit Antikörpern gegen Tau und Amyloid$\beta$ zu reduzieren. In den letzten Jahren sind jedoch Möglichkeiten entstanden, direkt auf die Entstehung dieser Proteinprodukte einzuwirken. Dabei werden AntisenseOligonukleotide (ASO) eingesetzt, die die
Proteintranslation pathologischer AlzheimerGene hemmen und damit frühzeitig auf die Krankheitsentwicklung Einfluss nehmen. Unser Beitrag fasst den aktuellen Stand der Entwicklung ASO-basierter Therapiestrategien bei der Alzheimer-Krankheit zusammen.

Schlüsselwörter

Gentherapie · Antikörpertherapie · AntisenseOligonukleotide $\cdot$ Amyloid- $\beta$ - Tau

\section{Gene-specific treatment approaches in Alzheimer's disease and other tauopathies}

\section{Abstract}

The pathological hallmarks of Alzheimer's disease are aggregation and accumulation of amyloid- $\beta$ and tau proteins. So far most interventional studies have focused on the removal of the toxic protein products, such as antibody-based immunotherapies targeted against amyloid- $\beta$ and tau proteins; however, the development of gene therapies targeting gene products involved in the disease has opened up new therapeutic strategies to reduce the development of toxic protein aggregates by inhibiting the translation of pathological Alzheimer genes using antisense oligonucleotides (ASO). This has a timely influence on development of the disease. This article gives an overview of new advances in ASO-based treatment strategies for Alzheimer's disease.

\section{Keywords}

Gene therapy - Antibody therapy · Antisense oligonucleotides · Amyloid beta · Tau von $\mathrm{A} \beta 1-42$ so wirksam reduziert werden kann (• Abb. 1B).

\section{MAPT}

Der Hauptbestandteil der neurofibrillären Tangles bei der AD ist hyperphosphoryliertes und aggregiertes Protein Tau. Die Ausprägung der Demenz hängt stark von der Last der neurofibrillären Tangles ab [19]. Zudem weisen AD-Patienten pathologisch erhöhte Tau-Werte im Liquor auf. Protein Tau wird von dem Mikrotubuliassoziierten-Protein-Tau-Gen (MAPT) auf Chromosom 17 kodiert. Mehr als 30 Mutationen im MAPTGen führen zu einer der AD ähnlichen Tauopathie. MAPT-Mutationen rufen in aller Regel eine frontotemporale Demenz hervor, lediglich die p.R406W-Mutation wurde auch in Familien mit Alzheimer-
Krankheit beschrieben [23]. Ein weiteres Indiz für die Relevanz der Tauopathie bei der $\mathrm{AD}$ ist die Beobachtung, dass ein MAPT-KO („knock-out“) im APPMausmodell eine Abschwächung der klinischen Symptomatik bewirkt [22].

Vor diesem Hintergrund wurden ASO-basierte Therapieansätze entwickelt, die auf eine Expressionsreduktion des Tau-Proteins abzielen. Präklinisch wurden ASOs entwickelt, die in Mäusen mit Expression eines humanen, mutierten MAPT-Gens (P301S-Tau) selektiv an die humane Tau-mRNA binden, welche RNAse-H-vermittelt abgebaut wird, wodurch weniger humanes Tau-Protein entsteht (• Abb. 1A). Nach intrathekaler Gabe des ASO kam es in P301S-Taumutanten Mäuse zu einer verminderten Bildung neuer Tau-Einschlüsse sowie deren Propagation, einem Rückgang 
der bereits vorhandenen Tau-Pathologie sowie zur Abnahme der Neurodegeneration im Hippokampus und $\mathrm{zu}$ einer Verlängerung des Überlebens. Auch in nichthumanen Primaten bewirkte das ASO nach intrathekaler Verabreichung eine Reduktion der Tau-Expression im Gehirn, Rückenmark und Liquor [7].

Aufgrund dieser vielversprechenden Daten testet Ionis Pharamceuticals Inc. aktuell ein entsprechendes ASO (BIIB080, ISIS 814907; IONIS-MAPTRx) in einer klinischen Phase-I/IIStudie (NCT03186989) bei frühsymptomatischen Alzheimer-Patienten. Es gibt bisher keine Zwischenergebnisse der Studie, das Studienende wird auf Anfang 2020 veranschlagt.

\section{》) Mithilfe von Exon-Skipping kann die Expression von 4R-Tau verringert werden}

Ein alternativer Ansatz, die Tau-Pathologie mithilfe der ASO-Technologie abzumildern, besteht darin, das Spleißen der Tau-Prä-mRNA in verschiedene Proteinisoformen zu beeinflussen. Je nachdem, ob das Exon 10 des Proteins Tau abgeschrieben wird, kommt es zur Produktion von Drei- oder Vier-Repeat-Tau (3R- und 4R-Tau). Normalerweise werden 3R-Tau und 4R-Tau zu gleichen Anteilen exprimiert, während es bei sporadischen Tauopathien wie der frontotemporalen Demenz mit Parkinsonismus oder progressiver supranukleärer Blickparese (PSP) zu einer erhöhten Expression von 4R-Tau kommt, das als besonders toxisch gilt. Die N279K-Spleißmutation in Exon 10 des MAPT-Gens führt $\mathrm{zu}$ einer $4 \mathrm{R}$-Tau-Überexpression (FTDP-17). Schoch et al. entwickelten ein ASO, das durch Exon-Skipping einen Ausschluss von Exon 10 aus der fertigen mRNA bewirkt und nach intrathekaler Applikation die Expression von 4RTau im Zentralnervensystem (ZNS) von N279K-Tau-mutanten Mäusen stabilverringert, ohne dabei die Gesamtlevel von Tau zu beeinflussen, was in der Langzeittherapie den aus einer Tau-Insuffizienz resultierenden Nebenwirkungen vorbeugen würde ([26]; - Abb. 1B). Es ist daher zu erwarten, dass diese ASO-Stra- tegie („4R to 3R splicing“) zeitnah bei Patienten mit diversen Tauopathien in klinischen Studien getestet wird.

Insgesamt stellt die Abmilderung der Tau-Pathologie somit nicht nur für die $\mathrm{AD}$, sondern auch für andere Tauopathien wie der kortikobasalen Degeneration, der progressiven supranukleären Blickparese, der Silberkornkrankheit und der frontotemporalen Demenz einen vielversprechenden Therapieansatz dar.

\section{APOE \&4}

Ein weiterer vielversprechender therapeutischer Ansatzpunkt ist das APOE- $\varepsilon 4$ Gen, der häufigste Risikofaktor der Lateonset-Alzheimer-Krankheit. ApolipoproteinE kodiert ein Glykoprotein, das für den Transport von Cholesterin und anderen Lipiden wichtig ist und maßgeblich an Nervenwachstum und -regeneration beteiligt ist. Es bestehen drei APOE-Allelvarianten, nämlich $A P O E \varepsilon 2$, $\varepsilon 3$ und $\varepsilon 4$, die für ApoE2, ApoE3 und ApoE4 kodieren. Heterozygote $A P O E-\varepsilon 4$-Träger haben ein bis zu 3-fach erhöhtes Risiko an Early-onset- oder Late-onset-Alzheimer-Krankheit zu erkranken, während homozygote $A P O E-\varepsilon 4$-Träger ein bis zu 15-fach erhöhtes Risiko haben [5].

\section{I) Durch Hemmung des Spleißens von Exon 19 behält ApoER2 seine volle Länge}

ApoE wirkt sich negativ auf den Abbau von Amyloid- $\beta$ aus und führt zur vermehrten Bildung von Amyloid- $\beta$ aus APP [6]. Unter anderem ist der ApolipoproteinE-Rezeptor 2 (ApoER2) für diesen Mechanismus verantwortlich, der bei der Alzheimer-Krankheit hauptsächlich in einer verkürzten Isoform vorliegt, die durch alternatives Spleißen am Exon19 entsteht. ApoER2 ist für die synaptische Übertragung und Langzeitpotenzierung wichtig, während die verkürzte Isoform einen negativen Effekt auf die zelluläre Aktivierung und Amyloidpathologie hat. Daher wurden ASO-Therapien untersucht, die an ApoER2-Sequenzen binden, die indirekt über SRSF1 („serine/ arginine-rich splicing factor 1 “) das Spleißen von Exon 19 hemmen und zur ver- mehrten Bildung von ApoER2 in seiner vollen Länge führen und damit dessen normale Rezeptorfunktion und Signalübertragung erhalten ([13]; • Abb. 1B). In einer präklinischen Studie konnte gezeigt werden, dass dies zur Verbesserung der synaptischen Funktion und kognitiven Leistung von APP-transgenen Mäusen führte. Dies hätte weitreichende Folgen da $A p o E$ \& 4 die höchste Prävalenz als Risikofaktor für die Alzheimer-Krankheit hat und bis zu 40-65\% der Patienten Apo- $\varepsilon 4$-Träger sind.

\section{Fazit für die Praxis}

\section{- Auf die Reduktion der Amyloid- $\beta$ - und der Tau-Pathologie abzielende Antisense-Oligonukleotide(ASO)- Strategien stellen vielversprechen- de Ansätze für die Behandlung genetisch bedingter, aber auch spo- radischer Alzheimer-Krankheit(AD)- Formen und Tauopathien dar. \\ - Angesichts der ernüchternden Ergeb- nisse der gegen Amyloid- $\beta$ gerichte- ten Immunisierungstherapien stellt sich grundsätzlich die Frage, ob die Amyloid- $\beta$-Pathologie im Stadium der manifesten Demenz das beste therapeutische Target ist. \\ - Die erste genspezifische ASO-The- rapie bei AD hat die Reduktion der Tau-Pathologie zum Ziel und befindet sich aktuell in Phase I/II der klinischen Testung.}

\section{Korrespondenzadresse}

\section{Dr. E. Feneberg}

Nuffield Department of Clinical Neurosciences, University of Oxford, Level 6, West Wing, John Radcliffe Hospital OX3 9DU Oxford, Großbritannien emily.feneberg@ndcn.ox.ac.uk

Funding. Open Access funding provided by Projekt DEAL.

\section{Einhaltung ethischer Richtlinien}

Interessenkonflikt. E. Feneberg und M. Otto geben an, dass kein Interessenkonflikt besteht.

Für diesen Beitrag wurden von den Autoren keine Studien an Menschen oder Tieren durchgeführt. Für die aufgeführten Studien gelten die jeweils dort angegebenen ethischen Richtlinien. 
Open Access. Dieser Artikel wird unter der Creative Commons Namensnennung 4.0 International Lizenz veröffentlicht, welche die Nutzung, Vervielfältigung, Bearbeitung, Verbreitung und Wiedergabe in jeglichem Medium und Format erlaubt, sofern Sie den/die ursprünglichen Autor(en) und die Quelle ordnungsgemäß nennen, einen Link zur Creative Commons Lizenz beifügen und angeben, ob Änderungen vorgenommen wurden.

Die in diesem Artikel enthaltenen Bilder und sonstiges Drittmaterial unterliegen ebenfalls der genannten Creative Commons Lizenz, sofern sich aus der Abbildungslegende nichts anderes ergibt. Sofern das betreffende Material nicht unter der genannten Creative Commons Lizenz steht und die betreffende Handlung nicht nach gesetzlichen Vorschriften erlaubt ist, ist für die oben aufgeführten Weiterverwendungen des Materials die Einwilligung des jeweiligen Rechteinhabers einzuholen.

Weitere Details zur Lizenz entnehmen Sie bitte der Lizenzinformation auf http://creativecommons.org/ licenses/by/4.0/deed.de.

\section{Literatur}

1. Biogen (2019) Biogen plans regulatory filing for aducanumab in Alzheimer's disease based on new analysis of larger dataset from phase 3 studies. https://investors.biogen.com/news-releases/ news-release-details/biogen-plans-regulatoryfiling-aducanumab-alzheimers-disease. Zugegriffen: 22.10.2019

2. Braak H, Braak E (1991) Neuropathological stageing of Alzheimer-related changes. Acta Neuropathol 82:239-259

3. Chang JL, Hinrich AJ, Roman Betal (2018) Targeting amyloid-beta precursor protein, APP, splicing with antisense oligonucleotides reduces toxic amyloidbeta production. Mol Ther 26:1539-1551

4. Chartier-Harlin MC, Crawford F, Houlden $\mathrm{H}$ et al (1991) Early-onset Alzheimer's disease caused by mutations at codon 717 of the beta-amyloid precursor protein gene. Nature 353:844-846

5. Corder EH, Saunders AM, Strittmatter WJ et al (1993) Gene dose of apolipoprotein E type 4 allele and the risk of Alzheimer's disease in late onset families. Science 261:921-923

6. Deane R, Sagare A, Hamm K et al (2008) apoE isoform-specific disruption of amyloid beta peptide clearance from mouse brain. J Clin Invest 118:4002-4013

7. DeVos SL, Miller RL, Schoch KM et al (2017) Tau reduction prevents neuronal loss and reverses pathological tau deposition and seeding in mice with tauopathy. Sci Transl Med 9(374):eaag481. https://doi.org/10.1126/scitranslmed.aag0481

8. DGN (2016) DGN Leitlinien. https://www.dgn.org/ leitlinien/3176-leitlinie-diagnose-und-therapievon-demenzen-2016\#therapie. Zugegriffen: 28.11.2019

9. Doody RS, Thomas RG, Farlow M et al (2014) Phase 3 trials of solanezumab for mild-to-moderate Alzheimer's disease. N Engl J Med 370:311-321

10. Erickson MA, Niehoff ML, Farr SA et al (2012) Peripheral administration of antisense oligonucleotides targeting the amyloid-beta protein precursor reverses AbetaPP and LRP-1 overexpression in the aged SAMP8 mouse brain. J Alzheimers Dis 28:951-960
11. Farr SA, Erickson MA, Niehoff ML et al (2014) Central and peripheral administration of antisense oligonucleotide targeting amyloid-beta protein precursor improves learning and memory and reduces neuroinflammatory cytokines in Tg2576 (AbetaPPswe) mice. J Alzheimers Dis 40:1005-1016

12. Haass C, Levin J (2019) Did Alzheimer research fail entirely? : failure of amyloid-based clinical studies. Nervenarzt 90:884-890

13. Hinrich AJ, Jodelka FM, Chang JLet al (2016) Therapeutic correction of ApoER2 splicing in Alzheimer's disease mice using antisense oligonucleotides. EMBO Mol Med 8:328-345

14. Jonsson T, Atwal JK, Steinberg S et al (2012) A mutation in APP protects against Alzheimer's disease and age-related cognitive decline. Nature 488:96-99

15. Kang J, Lemaire HG, Unterbeck A et al (1987) The precursor of Alzheimer's disease amyloid A4 protein resembles a cell-surface receptor. Nature 325:733-736

16. Kumar VB, Farr SA, Flood JF et al (2000) Sitedirected antisense oligonucleotide decreases the expression of amyloid precursor protein and reverses deficits in learning and memory in aged SAMP8 mice. Peptides 21:1769-1775

17. Laver K, Dyer S, Whitehead C et al (2016) Interventions to delay functional decline in people with dementia: a systematic review of systematic reviews. BMJ Open 6:e10767

18. Naslund J, Haroutunian V, Mohs R et al (2000) Correlation between elevated levels of amyloid beta-peptide in the brain and cognitive decline. JAMA 283:1571-1577

19. Nelson PT, Alafuzoff I, Bigio EH et al (2012) Correlation of Alzheimer disease neuropathologic changes with cognitive status: a review of the literature. J Neuropathol Exp Neurol 71:362-381

20. NovakP, SchmidtR, Kontsekova Eetal (2017) Safety and immunogenicity of the tau vaccine AADvac1 in patients with Alzheimer's disease: a randomised, double-blind, placebo-controlled, phase 1 trial. Lancet Neurol 16:123-134

21. Novak P, Zilka N, Zilkova M et al (2019) AADvac1, an active immunotherapy for Alzheimer's disease and non Alzheimer tauopathies: an overview of preclinical and clinical development. J Prev Alzheimers Dis 6:63-69

22. Roberson ED, Scearce-Levie K, Palop JJ et al (2007) Reducing endogenous tau ameliorates amyloid ß-induced deficits in an alzheimer's disease mouse model. Science 316(5825):750-754. https:// science.sciencemag.org/content/316/5825/750. full

23. Rademakers R, Dermaut B, Peeters K et al (2003) Tau (MAPT) mutation Arg406Trp presenting clinically with Alzheimer disease does not share a common founder in Western Europe. Hum Mutat 22:409-411

24. Salloway S, Sperling R, Fox NC et al (2014) Two phase 3 trials of bapineuzumab in mildto-moderate Alzheimer's disease. N Engl J Med 370:322-333

25. Salloway S, Sperling R, Gilman Setal (2009) A phase 2 multiple ascending dose trial of bapineuzumab in mild to moderate Alzheimer disease. Neurology 73:2061-2070

26. Schoch KM, Devos SL, Miller RL et al (2016) Increased 4R-tau induces pathological changes in a human-tau mouse model. Neuron 90:941-947

27. Selkoe DJ, Hardy J (2016) The amyloid hypothesis of Alzheimer's disease at 25 years. EMBO Mol Med 8:595-608
28. St. George-Hyslop PH, Tanzi RE, Polinsky RJ et al (1987) The genetic defect causing familial Alzheimer's disease maps on chromosome 21. Science 235:885-890 